\title{
Avaliação de Sintomas Psicológicos no Transtorno da Acumulação: Um Estudo de Caso
}

\author{
Diego Rafael Schmidt ${ }^{1}$, Cristina Pilla Della Méa ${ }^{1}$ \\ Faculdade Meridional (IMED), Passo Fundo-RS, Brasil
}

RESUMO

O Transtorno da Acumulação (TA) caracteriza-se pela aquisição de itens desnecessários, dificuldade em se desfazer de objetos e a desorganização do ambiente de convívio. O estudo de caso analisa as características de personalidade e os sintomas psicológicos comórbidos em uma paciente com diagnóstico de TA. Para o levantamento de dados, utilizou-se uma entrevista semiestruturada, o Questionário dos Esquemas de Young (QEY-S2), o Inventário dos Estilos Parentais de Young (IEP), o Inventário de Depressão e Ansiedade de Beck (BDI-II e BAI, respectivamente), o Inventário de Sintomas de Stress de Lipp (ISSL) e o Método de Rorschach. Nos resultados, observou-se a presença de Esquemas Iniciais Desadaptativos (EIDs) pertencentes ao domínio de desconexão e rejeição, sugerindo uma conexão entre os sintomas do TA e experiências emocionais negativas vivenciadas pela paciente durante a infância. Futuramente, recomenda-se a realização de novos estudos com delineamento transversal no intuito de substanciar os resultados obtidos pela presente pesquisa.

Palavras-chave: transtorno da acumulação, depressão, ansiedade, esquemas iniciais desadaptativos.

\section{ABSTRACT - Psychological symptom evaluation in accumulation disorder: A case study}

Accumulation disorder (AD) is characterized by the acquisition of unnecessary items, difficulty in disposing objects, and disorganization of the convivial environment. The case study analyzes personality characteristics and comorbid psychological symptoms in a patient diagnosed with AD. For data collection, a semi-structured interview was used, the Young Schema Questionnaire (YSQ-S2), the Young Parenting Styles Inventory (PSI), the Beck Depression Inventory and the Beck Anxiety Inventory (BDI-II and BAI, respectively), the Lipp Stress Symptoms Inventory (LSSI), and the Rorschach Method. In the results, we observed the presence of Early Maladaptive Schemas (EMS) belonging to the disconnection and rejection domain, suggesting a connection between AD symptoms and negative emotional experiences experienced by the patient during childhood. In the future, new studies with a cross-sectional design are recommended in order to substantiate the results obtained by the present research.

Keywords: Accumulation disorder; Depression; Anxiety; Early Maladaptive Schemas.

\section{RESUMEN - Evaluación de síntomas psicológicos en trastorno de acumulación: Estudio de un caso}

El trastorno de acumulación (TA) se caracteriza por adquisición de artículos desnecesarios, dificultad de deshacerse de objetos y desorganización en el ambiente de convivencia. El estudio de caso analiza las características de personalidad y los síntomas psicológicos comórbidos en un paciente con diagnóstico de TA. Para el levantamiento de datos se ha utilizado una entrevista semiestructurada, el cuestionario de los Esquemas de Young (QEY-S2), el Inventario de los Estilos Parentales de Young (IEP), el Inventario de Depresión y Ansiedad de Beck (BDI-II e BAI, respectivamente) el Inventario de Síntomas de Stress de Lipp (ISSL) y el Método de Rorschach. En los resultados, se ha observado presencia de Esquemas iniciales desadaptativos (EIDs) pertenecientes al dominio de desconexión y rechazo, sugiriendo una conexión entre los síntomas del TA y experiencias emocionales negativas vividas por el paciente durante la infancia. Futuramente, se recomienda la realización de nuevos estudios con delineamiento transversal con el fin de sustanciar los resultados obtenidos por esta investigación.

Palabras clave: Trastorno de acumulación; Depresión; Ansiedad; Esquemas iniciales desadaptativos.

\section{Introdução}

O Transtorno da Acumulação (TA) consiste na aquisição excessiva de itens desnecessários e na dificuldade persistente em desfazer-se dos objetos, o que acarreta ampla desorganização no ambiente de convívio do paciente (American Psychiatric Association, APA, 2014;
Araújo \& Lotufo Neto, 2014). Geralmente, os itens são recolhidos e armazenados de maneira exagerada e intencional, independentemente da sua real utilidade ou valia (Frost \& Steketee, 2014), comprometendo e obstruindo o espaço físico na residência do indivíduo (Ayers, Najmi, Mayes, \& Dozier, 2014). Quando se depara com a possibilidade de desfazer-se das posses, a pessoa que acumula 
normalmente experimenta emoções negativas de forma desagradavelmente intensa (Kress, Stargell, Zoldan, \& Paylo, 2016), pois acredita fortemente que necessitará dos itens coletados no futuro (Mathews, 2014; Schmidt, Della Méa, \& Wagner, 2014).

Nesse sentido, conforme Brakoulias e Milicevic (2015), a gravidade dos sintomas associados ao TA compromete a execução das atividades diárias básicas e interfere consideravelmente na qualidade de vida do paciente (Schmidt et al., 2014), prejudicando de maneira substancial a sua adaptação (Fontenelle \& Grant, 2014). Os sintomas acabam ocasionando sofrimento clinicamente significativo ao indivíduo e a seus familiares (APA, 2014), provocando sérios prejuízos em diversas áreas, como o contexto ocupacional, social e interpessoal (Thompson, Cruz, Mataix-Cols, \& Onwumere, 2016). Um estudo desenvolvido por Frías-Ibáñez, Palma-Sevillano, BarónFernández, Bernáldez-Fernández e Aluco-Sánchez (2014) sugere ainda que, muitas vezes, os indivíduos com TA não reconhecem a intensidade dos prejuízos decorrentes da própria psicopatologia e, consequentemente, encontram dificuldades em aderir às terapêuticas propostas (Mathews, 2014).

De acordo com o Manual diagnóstico e estatístico de transtornos mentais, DSM-5 (APA, 2014), estima-se a prevalência do transtorno em aproximadamente 2 a $6 \%$ da população geral, sendo mais frequentemente encontrado em adultos solteiros, na meia idade e do gênero masculino (Albert et al., 2015). Entretanto, Muroff e Underwood (2006) observam que, nas amostras clínicas, o TA é mais prevalente em mulheres e pouco comum nas crianças. Normalmente, o surgimento dos sintomas ocorre no final da adolescência ou no começo da idade adulta, de modo que o início precoce da patologia habitualmente indica prognóstico pouco favorável (APA, 2014). Em relação à etiologia do TA, Mataix-Cols (2014) presume que fatores genéticos, cognitivos e/ou ambientais possam estar relacionados às causas do transtorno, caracterizando-o como um quadro preocupante e de sintomatologia predominantemente refratária (Ayers et al., 2014; Muroff \& Underwood, 2016).

Segundo Wheaton (2016), a gravidade dos sintomas de acumulação pode estar principal e diretamente associada a prejuízos nas funções executivas, como tomada de decisão, atenção e memória, as quais ocasionam problemas nos processos psicológicos de organização e categorização (Grisham \& Baldwin, 2015). Por isso, pacientes com TA podem manifestar uma tendência à procrastinação e, em alguns casos, ao perfeccionismo extremo na realização de suas tarefas (Ayers et al., 2014). Da mesma forma, pesquisas recentes de Timpano, Shaw, Cougle e Fitch (2014) e Shaw, Timpano, Steketee, Tolin e Frost (2015) demonstraram que a falta de habilidade em tolerar emoções desagradavelmente intensas pode induzir o paciente a manifestar condutas evitativas em diversas situações. Não raro, o curso do TA pode ocorrer concomitantemente a outros transtornos mentais COmórbidos, como depressão (Ayers et al., 2014; Muroff, Steketee, Frost, \& Tolin, 2014), ansiedade (Brakoulias \& Milicevic, 2015), transtorno de déficit de atenção com hiperatividade, transtornos da personalidade e esquizofrenia, entre outros (Frost \& Steketee, 2014).

No que se refere às principais intervenções terapêuticas estudadas para o tratamento do quadro, pesquisas realizadas por Brakoulias, Eslick e Starcevic (2015), Kress, Stargell, Zoldan, e Paylo (2016) e Muroff e Underwood (2016) destacam a psicofarmacologia e a terapia cognitivo-comportamental (TCC), que aborda os aspectos comportamentais, cognitivos e emocionais do indivíduo para o abatimento dos sintomas. Segundo o modelo cognitivo do TA, são as crenças do paciente que mantêm a sua sintomatologia (Schmidt et al., 2014), de modo que a psicoterapia cognitivo-comportamental consista em reduzir o recolhimento descontrolado dos itens (Wheaton, 2016), bem como promover a organização e a desobstrução do ambiente de convívio (Muroff et al., 2014). Juntamente com a exposição gradual ao descarte dos objetos coletados, realiza-se o processo de reestruturação cognitiva (Tolin, Frost, Steketee, \& Muroff, 2015) no intuito de identificar os padrões de pensamento disfuncionais que propiciam a acumulação excessiva e impedem o descarte (Albert et al., 2015; Frost \& Steketee, 2014).

Muitas vezes, além da reestruturação cognitiva em psicoterapia (Wheaton, 2016), torna-se necessário o trabalho com as experiências negativas de vida e os Esquemas Iniciais Desadaptativos (EIDs), que, de acordo com Young, Klosko e Weishaar (2008), correspondem às estruturas cognitivas relacionadas à maneira como o indivíduo percebe a realidade. De uma maneira geral, os EIDs demonstram significativa associação com o surgimento e a manutenção de psicopatologias como o TA (Schmidt et al., 2014), fazendo-se necessária a avaliação de aspectos que possam interferir diretamente nas características clínicas do transtorno.

Nesse sentido, faz-se importante o desenvolvimento de pesquisas relacionadas ao diagnóstico de TA com o propósito de identificar a presença de eventuais comorbidades, o que pode facilitar a adesão e promover o desenvolvimento de abordagens efetivas para o tratamento do transtorno. Considerando a alta incidência de comorbidades e os aspectos de personalidade frequentemente descritos pela literatura como associados ao TA, foram escolhidos instrumentos específicos que identificassem a presença e a intensidade dos principais sintomas comórbidos relacionados a esse transtorno. Num primeiro momento, uma das hipóteses levantadas refere-se à existência de sintomas de ansiedade, depressão e estresse concomitantemente ao diagnóstico do TA. Semelhantemente, suspeitou-se da presença de EIDs clinicamente relevantes, bem como problemas de relacionamento, o que também justifica a escolha dos instrumentos utilizados neste estudo de caso. 
Assim, o presente trabalho tem como objetivo avaliar as características de personalidade e os sintomas psicológicos comórbidos apresentados por uma paciente com diagnóstico de transtorno da acumulação de animais e histórico de coleta de objetos em geral.

\section{Método}

$\mathrm{O}$ artigo consiste em um estudo de caso baseado num modelo qualitativo de pesquisa empírica (Yin, 2015) que visa obter informações sobre o TA, especialmente no que se refere aos sintomas psicológicos comórbidos e às características de personalidade apresentadas pela paciente. Participou do estudo uma paciente com diagnóstico de TA, indicada por um psicólogo clínico que atuava em consultório particular de psicoterapia localizado no interior do estado do Rio Grande do Sul.

Para fins de viabilidade do estudo, foram contatados psicólogos clínicos localizados no interior do estado do Rio Grande do Sul, apresentando a proposta de pesquisa para, após a aprovação do projeto pelo Comitê de Ética em Pesquisa (CEP) de uma instituição superior localizada no interior desse estado, obter a indicação de possíveis participantes. Para a seleção do sujeito de pesquisa, incluíram-se pacientes maiores de 18 anos e com diagnóstico de TA. Apenas um dos profissionais identificou entre os seus clientes um sujeito com as características do transtorno. O psicólogo clínico disponibilizou-se a prosseguir com a pesquisa, considerando os termos e as condições éticas estabelecidos pela Resolução n ${ }^{\circ}$. 466/12 do Conselho Nacional de Saúde (CNS).

Após a aprovação do projeto pelo CEP, sob parecer $\mathrm{n}^{\mathrm{o}}$. 1.176.030, o pesquisador efetuou contato telefônico com a paciente em questão para agendar um horário pessoalmente e informar o objetivo do trabalho, realizando o convite para a pesquisa. Desde o contato inicial, a paciente foi orientada acerca das ressalvas éticas relacionadas ao estudo e o uso das informações disponibilizadas, bem como o seu caráter sigiloso. Reforçou-se, ainda, que, caso a paciente decidisse, poderia optar por abandonar a pesquisa a qualquer momento sem acarretar quaisquer prejuízos a si mesma ou ao seu processo de psicoterapia.

Mediante a assinatura do Termo de Consentimento Livre e Esclarecido (TCLE), conforme a Resolução n ${ }^{\circ}$. 466/12, deu-se início à aplicação da entrevista semiestruturada e dos demais instrumentos, que ocorreram em dois encontros com duração aproximada de 1 hora e 30 minutos, realizados na residência da paciente. No momento em que a pesquisa foi realizada, a participante encontrava-se em atendimento psicológico e frequentava à terceira sessão de psicoterapia cognitivo-comportamental. Segundo relatos da paciente, a busca pelo atendimento psicológico ocorreu pela necessidade de abordar algumas questões relacionadas à sua história de vida.
Além da entrevista semiestruturada elaborada pelos autores, para a coleta de dados do presente estudo foram utilizados o Questionário dos Esquemas de Young - versão reduzida (YSQ-S2), o Inventário dos Estilos Parentais de Young - versão reduzida (IEP), o Inventário de Depressão e de Ansiedade de Beck (BDI-II e BAI, respectivamente), o Inventário de Sintomas de Stress de Lipp (ISSL) e o Método de Rorschach, no sistema compreensivo, conforme descrição abaixo:

1. Entrevista semiestruturada: consiste em um instrumento elaborado pelos autores com base nos critérios diagnósticos do TA (APA, 2014), que tem por finalidade levantar informações acerca do perfil sociodemográfico da paciente e, especialmente, da sintomatologia apresentada.

2. Questionário dos Esquemas de Young, versão reduzida (QEY-S2): trata-se de um questionário desenvolvido por Young et al. (2008) e validado para a população brasileira por Cazassa e Oliveira (2012), que visa avaliar EIDs do paciente. A ferramenta é composta por 75 itens preenchidos em escala Likert que indicam a presença de EIDs.

3. Inventário dos Estilos Parentais de Young, versão reduzida (IEP; Valentini, Alchieri, \& Laros, 2013): tem por objetivo avaliar a relação entre os estilos parentais, ou seja, os padrões de interação entre pais-filhos e os EIDs. Por meio de uma escala Likert, o instrumento com 49 itens procura identificar os estilos de interação entre paciente e os cuidadores (Valentini \& Alchieri, 2009), que também se relacionam com o desenvolvimento e a manutenção de psicopatologias (Young et al., 2008).

4. Inventário de Depressão de Beck (BDI-II) e Inventário de Ansiedade de Beck (BAI) (Cunha, 2001): o BDI-II e o BAI consistem em instrumentos com 21 itens que avaliam os sintomas depressivos e ansiosos, respectivamente, por meio de medidas de autorrelato, classificando-os no que se refere à sua presença e intensidade.

5. Inventário de Sintomas de Stress de Lipp (ISSL): trata-se de um instrumento desenvolvido por Lipp (2000), com 56 itens que avaliam a existência de sintomas de estresse em adultos, classificando-os quanto à sua característica predominantemente somática ou psicológica.

6. O Método de Rorschach, no Sistema Compreensivo: possibilita a investigação de traços de psicopatologia (Nascimento, 2010), bem como aspectos cognitivos (Hisatugo \& Yazigi, 2014), relacionais (Nascimento, 2012) e afetivos do sujeito avaliado, auxiliando o psicólogo na compreensão do funcionamento da personalidade (Mihura, Meyer, Dumitrascu, \& Bombel, 2013).

Por fim, os resultados dos testes foram interpretados de acordo com os seus respectivos manuais e, em segui$\mathrm{da}$, analisados clinicamente para integrar as informações obtidas com a história pregressa e a sintomatologia apresentada pela participante, resultando no presente relatório de pesquisa. 


\section{Resultados e Discussão}

\section{Descrição do Caso Clínico}

Martha (nome fictício), 50 anos, ensino médio completo, é divorciada e tem uma filha. Atualmente, é proprietária de uma empresa localizada no interior do estado do Rio Grande do Sul, na qual desempenha atividades da área administrativa. A paciente foi recrutada de um consultório particular de psicoterapia no interior do estado por meio de indicação pelas características de TA que manifesta, isto é, o acúmulo de animais. Durante a entrevista, demonstrou-se colaborativa e disposta em preencher os instrumentos, não indicando qualquer desconforto em relação à pesquisa.

\section{Sintomatologia}

No que se refere à sintomatologia, Martha atualmente reside em um apartamento com a filha, no qual também convivem com nove gatos. Ela relata que adotou o primeiro animal de estimação há cerca de 17 anos, a pedido da filha, e, depois disso, passou a sentir-se exageradamente incomodada ao ver outros bichos em situação de risco. Segundo a participante, os sentimentos de piedade e incômodo ocorrem porque "o bicho não tem como se defender. Se ele estiver quebrado, dificilmente alguém vai recolher [...]. Mas eu tenho pena, o sofrimento deles é algo que me incomoda bastante". Muitas vezes, mobilizada pela percepção de que animais estavam em situação de vulnerabilidade, Martha os levou para a sua casa para proporcionar cuidado, tendo rapidamente se afeiçoado a eles. Relatou, inclusive, que, por muito tempo, levou consigo, dentro da bolsa, ração e água, no intuito de alimentar os animais da rua que encontrasse.

Em relação aos animais recolhidos, refere que, se pudesse, teria muitos outros, porém, no momento, não tem condições físicas, emocionais, nem financeiras para prover tal cuidado (sic.). Embora se sinta exausta por causa dos gatos e por privar-se dos momentos de lazer que tinha anteriormente, Martha acredita que ninguém proporcionará o mesmo tipo de cuidado que o seu, o que dificulta a doação dos bichos. "Digamos [que], daqui a pouco eu morra, quem é que vai cuidar? Não tem quem cuide e não vão cuidar como eu. Eles [os animais] vão sofrer". Semelhantemente, para ela, a recompensa emocional proporcionada pelo animal é grande: "eu comecei a ver que o animal te dá um retorno muito grande: ele tá sempre ali do teu lado. Da maneira dele, ele te dá carinho, te dá atenção. Ele não te questiona e ele é dependente de ti... e eu acho que isso é o que te sensibiliza mais".

Com o tempo, o recolhimento dos animais acabou ocasionando significativa desorganização no ambiente de convívio de Martha e, por conseguinte, interferindo na execução das suas atividades diárias: "eu ando com um atraso na minha vida de uns três anos pra cá, que foi acumulando, acumulando, acumulando... Então, agora eu tô tentando aos poucos organizar de forma que fique mais fácil de, por exemplo, me organizar com roupa, com casa, com tudo". Continuamente, a limpeza do ambiente exige bastante do seu tempo, deixando-a cansada e com poucos horários para rotinas básicas, como o sono e o lazer. Antes, segundo ela, "eu fazia tudo assim, desde o início, meio e fim, isto né, nesta ordem, religiosamente, e hoje em dia eu não consigo fazer [...]. Eu tinha uma rotina que era tranquila e que agora a minha vida é bem mais tumultuada".

Por muito tempo, a paciente relata que se privou de várias atividades em virtude do cuidado dos animais (i.e., ler livros, estudar, assistir televisão, cuidar da casa, etc.), o que a deixa bastante irritada. "Mas eu me sinto, hoje, cansada, com o corpo pesado, eu não tenho mais a mesma rapidez, a mesma agilidade". O relato da paciente sugere que a desorganização decorrente do acúmulo excessivo a levou a diminuir o recolhimento dos animais: "Bom, isso já não acontece mais porque eu não tenho estrutura, nem física, nem financeira e nem emocional, eu não tenho mais. Porém, às vezes, eu peguei pensando em disponibilizar para a adoção, só que comigo isso não funciona. Eu me afeiçoo muito rapidamente e daí eu já começo a colocar muitos empecilhos nos possíveis pretendentes. Às vezes, nem aparece. No caso da maioria dos bichos que eu tenho, [para] muitos não apareceu pretendentes, mas os que apareceram, eu já comecei a achar "não vai cuidar direito', não vai isso, não vai aquilo...”.

Em relação ao princípio do acúmulo, Martha lembra-se que inicialmente guardava objetos em geral: "eu já fui muito apegada a coisas materiais; hoje em dia não sou mais [...]. Houve épocas em que eu guardava revistas, livros, papeizinhos... hoje em dia eu não guardo mais isso...". De acordo com o relato da paciente, a quantidade de objetos coletados naquela época não era significativa, contudo o tempo de permanência com tais itens era considerável, o que pode ser caracterizado como um indicativo inicial da acumulação.

Atualmente, no entanto, comenta que encontra relativa facilidade em desfazer-se de objetos, mas ainda costuma guardá-los com certa regularidade: "porque eu guardo pensando que eu vou utilizar. Mas não adianta, eu vejo que eu não utilizei e eu boto fora. Isso acontece com objetos, potes e coisas assim...”. Com relação à frequência com que isso ocorre, normalmente varia de dias ou meses, até anos, dependendo do caso, mas não causa nenhum sofrimento ou prejuízo (sic.). Entretanto, ao que parece, o recolhimento de objetos foi gradativamente substituído pelo de animais: "[antes] eu até me sensibilizava, mas eu não me envolvia. Eu tinha, digamos, uma certa frieza. E, no momento que eu peguei um, que eu adotei o primeiro, que aquele foi de livre e espontânea vontade, eu passei a ver outros. E daí aquilo começou a me incomodar". Ainda assim, Martha afirma que nunca sentiu culpa ou arrependimento depois de recolher os animais. 
Quando questionada acerca dos seus relacionamentos interpessoais, a participante descreve que evita manter vínculos de proximidade com familiares ou amigos e, frequentemente, prefere ficar sozinha a estar próxima de outras pessoas. "Eu não sinto falta disso. Eu não sinto falta de ter uma pessoa assim pra conversar [...]. Eu não procuro manter vínculo com ninguém. Se eu puder evitar, eu evito". De uma maneira geral, Martha considera difícil relacionar-se com pessoas em comparação aos animais. Para ela, "a pessoa é uma coisa muito complexa $[\ldots]$, normalmente, ela vai ter uma ou duas coisas que você vai gostar nela e muitas que tu não vai gostar, certo? E conviver com isso é ruim, é trabalhoso, não é muito bom. Ao passo que o bichinho, o bichinho não: ele vai ter sempre o mesmo comportamento...".

\section{Histórico Familiar}

No que se refere à origem da sua família materna, Martha explica que é descendente de poloneses refugiados da guerra que fixaram residência no interior do estado do Rio Grande do Sul. Quando questionada, conta que o seu avô materno misteriosamente desapareceu em uma estação de trem, deixando a avó grávida e com seis filhos sozinhos no local. Compadecido com a situação, um conhecido da família os teria abrigado em um paiol, onde permaneceram por bastante tempo. Segundo relatos da paciente, a avó era uma mulher agressiva e tinha problemas com álcool, o que fez com que seus filhos saíssem de casa para trabalhar. Na época, Sônia (nome fictício), a mãe da paciente, acabou em uma casa de meretrício, na qual conhecera Pedro (nome fictício), o pai da paciente, e com ele se envolveu. Pedro era de uma família com grande poder aquisitivo, caracterizada pela paciente como tradicional e conservadora, a qual desaprovava o relacionamento do casal, principalmente em razão da passagem de Sônia por uma casa de meretrício.

Mesmo com a censura da família, Pedro casou-se com Sônia e tiveram filhos, sendo Martha a mais velha de um total de quatro crianças concebidas pelo casal. A paciente descreve a relação dos seus pais como bastante complicada e minimamente afetiva. De acordo com a participante, Sônia tinha sérios problemas com álcool que ocasionavam mudanças no seu comportamento: "quando ela bebia, ela se transformava: ficava violenta, fazia nós sentir uma imensa vergonha porque gritava palavrão, tirava a roupa, ficava violenta, que tu não podia entrar dentro de casa, ela atirava o que tinha pela frente...”. Especialmente quando bebia, Sônia tinha explosões de raiva, fazendo com que, enquanto a mãe estivesse sob efeito do álcool, Martha ficasse boa parte do tempo fora de casa. "Ela tinha raiva de mim, ela tinha ódio, principalmente quando bebia [...]. Com os outros, ela não era tão violenta quanto era comigo [...]. Teve uma vez que ela foi agredir o pai e eu fui segurar ela e daí ela acertou uma facada no meu braço. Daí uma outra vez ela jogou uma frigideira de banha quente (ri), em cima de mim.
Outra vez, ela pegou e bateu com uma garrafa no meu braço e daí ficou inchado...”.

$\mathrm{O}$ alcoolismo de Sônia piorou significativamente com o tempo, o que fez com que Pedro procurasse constituir nova família. Para Martha, "o pai [...] sempre foi uma pessoa ausente, porque, na medida que [Sônia] começou a ter problema, ele simplesmente sumiu, e cada vez menos [...]. Também nunca foi uma pessoa muito carinhosa". O distanciamento do pai fez com que Martha se sentisse desamparada: "ele não se preocupava com os filhos, se tavam vivos, se tavam doentes, se não tavam, se iam pra escola, o que acontecia... não [...]. Na realidade, ele nos anulou do mundo dele". Consequentemente, Martha e os irmãos saíram de casa para afastarem-se de Sônia: "ela tava num nível tão grande de dependência que ela chegava falar sozinha, delirar, uma coisa assim... E nós, como a gente não tinha o apoio de ninguém, logo que começou a crescer, a gente procurou sair de casa".

Por fim, com o passar do tempo e o aumento do consumo de álcool, Sônia tornou-se descuidada em relação à própria alimentação e higiene: "Ela não se cuidava mais, não tomava mais banho, às vezes passava uma semana [...] bebendo, não comia...". Mais tarde, devido a ferimentos sérios decorrentes de uma queda, Martha foi chamada para levar Sônia ao hospital, despertando sentimentos de raiva na participante: "Mas eu fiquei com tamanha ira, tamanha ira, que eu disse 'Mas Deus, porque não morre pra parar de incomodar?'”. Acompanhada por Pedro e um vizinho, Martha ofereceu os cuidados necessários, mas Sônia, não estando em condições de sair, rejeitou o atendimento médico, e faleceu naquela noite, em um acidente doméstico. Desde então, Martha relata que sente culpa e remorso pela perda da mãe.

\section{Resultados da Avaliação Psicológica}

Os resultados dos instrumentos aplicados para avaliar sintomas comórbidos e as características de personalidade na paciente são, aqui, apresentados em três tabelas. No que se refere à descrição dos escores de ansiedade, depressão e estresse avaliados pelo BAI, BDI-II e o ISSL, as informações podem ser observadas na Tabela 1.

De acordo com os resultados obtidos pela aplicação dos instrumentos BAI, BDI-II e o ISSL, a participante não manifesta a presença de sintomatologia significativa de ansiedade, depressão e estresse, respectivamente. Contudo, a análise qualitativa do BDI-II indica a presença de escores maiores atribuídos aos itens do instrumento que se relacionam à falta de energia e à pouca vontade para desenvolver as atividades, os quais foram relatados pela paciente durante a entrevista. Em relação aos sintomas mencionados, Martha explica: "Mas eu me sinto, hoje, cansada, com o corpo pesado, eu não tenho mais a mesma rapidez, a mesma agilidade que eu tinha há algum tempo atrás [...]. O meu corpo já não responde da mesma forma".

Os EIDs avaliados pelo QEY-S2 podem ser analisados na Tabela 2. 
Tabela 1

Descrição dos Escores de Ansiedade, Depressão e Estresse

\begin{tabular}{lcc}
\hline \multicolumn{1}{c}{ Instrumento } & Escore & Nível \\
\hline Inventário de Ansiedade de Beck (BAI) & 5 & mínimo ou ausente \\
Inventário de Depressão de Beck (BDI-II) & 10 & mínimo ou ausente \\
Inventário de Sintomas de Stress de Lipp (ISSL) & 6 & mínimo ou ausente \\
\hline
\end{tabular}

Fonte. Primária (2015)

Tabela 2

Domínios Esquemáticos e EIDs pela Paciente no QEY-S2

\begin{tabular}{|c|c|c|}
\hline Domínios esquemáticos & EIDs & Média \\
\hline \multirow[t]{5}{*}{ Desconexão e rejeição } & Privação emocional & $5,40^{*}$ \\
\hline & Abandono & 1,00 \\
\hline & Desconfiança/Abuso & 1,40 \\
\hline & Isolamento social & $4,60^{*}$ \\
\hline & Defectividade/Vergonha & 1,00 \\
\hline \multirow[t]{4}{*}{ Autonomia e desempenho prejudicados } & Fracasso & 1,00 \\
\hline & Dependência/Incompetência & 2,00 \\
\hline & Vulnerabilidade ao dano e à doença & 1,00 \\
\hline & Emaranhamento/Self subdesenvolvido & 1,00 \\
\hline \multirow[t]{2}{*}{ Direcionamento para o outro } & Subjugação & 1,00 \\
\hline & Autossacrifício & $5,00^{*}$ \\
\hline \multirow[t]{2}{*}{ Supervigilância e inibição } & Inibição emocional & $4,00^{*}$ \\
\hline & Padrões inflexíveis/Postura crítica exagerada & 3,00 \\
\hline \multirow[t]{2}{*}{ Limites prejudicados } & Arrogo/Grandiosidade & 1,00 \\
\hline & Autocontrole/Autodisciplina insuficientes & 1,00 \\
\hline Total & & 34,00 \\
\hline
\end{tabular}

Fonte. Primária (2015). *Escores clinicamente relevantes

Os escores do QEY-S2 indicam a presença de esquemas de privação emocional $(m=5,40)$ e autossacrifício $(m=5,00)$. Segundo Van Genderen, Rijkeboer e Arntz (2012), o EID de privação emocional refere-se à expectativa manifestada pelo paciente de que as suas necessidades emocionais de afeto, empatia e proteção não serão atendidas. Normalmente, os sujeitos com esse EID sentem-se incompreendidos pelos outros (Farrell, Reiss, \& Shaw, 2014) e podem encontrar dificuldades em aceitar as suas necessidades afetivas como naturais e corretas (Wainer, Paim, Erdos, \& Andriola, 2016). Considerando o relato da participante, sugere-se que o comportamento agressivo da mãe e a ausência do pai possam estar associados ao EID de privação emocional. Em suas palavras, "na questão afeição deles, do pai ou da mãe, eu não lembro [...]. Se existiu, foi quando eu era muito criança e eu não tenho recordações disso".

No esquema de autossacrifício, contudo, o indivíduo demonstra preocupação excessiva pelas necessidades alheias, diferentemente das próprias, tornando-se demasiadamente empático e altruísta (Van Genderen et al., 2012). Os sujeitos com o esquema de autossacrifício apresentam um senso voluntário de responsabilidade em relação a terceiros que pode assumir característica exagerada, levando-os a experimentar sentimentos de culpa e ressentimento (Wainer et al., 2016). No caso de Martha, considerando que a paciente demonstra pouca necessidade de relacionamento interpessoal, sugere-se que o EID de autossacrifício também possa estar relacionado com o cuidado dos animais (i. e., "eu sou bem seletiva em ajudar as pessoas, não são todos [...]. Ao passo que bicho, da forma que tiver, eu não olho: eu ajudo" e "a pessoa, se fosse pegar, ela por mais amor que tivesse pelos animais, ela não faria o que eu faço. Não, jamais").

Semelhantemente, mas com menor ênfase, observa-se que os esquemas de isolamento social $(m=4,60)$ e inibição emocional $(m=4,00)$ também foram assinalados pela participante. No esquema de isolamento social, conforme o modelo de Young et al. (2008), o sujeito sente-se diferente dos demais e acredita não pertencer a nenhum 
grupo social, isolando-se do resto do mundo. Muitas vezes, pacientes com esse esquema sentem-se excluídos (Lopes \& Melo, 2014) e podem manifestar pouco ou nenhum interesse em realizar atividades sociais (Farrell et al., 2014), o que também foi relatado previamente por Martha. Segundo a participante, "são pouquíssimas as [pessoas] que eu tenho alguma intimidade além do estritamente social", fazendo com que ela desenvolvesse crenças específicas, como "eu não gosto de pessoas", "eu nunca tive muito bom relacionamento com pessoas da mesma espécie", "eu não procuro manter vínculo com ninguém" e, ainda, "eu sou diferente, completamente diferente [das outras pessoas]".

Já o EID de inibição emocional caracteriza-se pela restrição exagerada das emoções do indivíduo, ocasionando séria diminuição na sua espontaneidade e expressão afetiva (Lopes \& Melo, 2014). Trata-se de pessoas que costumam conter as suas emoções e temem a desaprovação dos outros, comportando-se de maneira demasiadamente controlada e inibida (Van Genderen et al., 2012). No caso de Martha, o EID de inibição emocional pode ser observado em diferentes crenças da participante, como "eu não sei expressar aquele carinho, como eu vejo muitas pessoas, um abraço, uma coisa mais assim... eu tenho uma dificuldade imensa, eu não sei fazer isso", "eu não costumo me abrir muito com as pessoas" e "eu não sou muito de afeições, sou uma pessoa bastante racional e bastante prática".

No que se refere aos esquemas cognitivos no TA, recentemente, um estudo desenvolvido por Rasmussen, Steketee, Silverman e Wilhelm (2013) avaliou a relação entre EIDs e o transtorno da personalidade esquizotípica em indivíduos com sintomas de TA. Aparentemente, os resultados sugerem que o comportamento de acumular e as dificuldades no descarte associam-se fortemente aos esquemas de inibição emocional, privação emocional e autossacrifício, respectivamente. Semelhantemente, na presente pesquisa, também se verificou a presença dos esquemas mencionados por Rasmussen et al. (2013), sugerindo que a aquisição excessiva de objetos ou animais e a dificuldade em desfazer-se de tais itens possa estar fortemente relacionada às experiências de privação material e problemas de apego durante a infância, assim como sugerem os estudos de Shaw, Witcraft e Timpano (2015).

No que se refere aos estilos parentais (IEP) apresentados na Tabela 3, verificou-se presença de escores elevados associados ao Fator I: desconexão e rejeição (escala materna: $m=3,61$; escala paterna: $m=3,39$ ), o qual se relaciona a características parentais de rejeição, instabilidade e negligência emocional. Cabe ressaltar, ainda, que os esquemas de privação emocional e isolamento social encontrados anteriormente no QEY-S2 também pertencem ao domínio esquemático de desconexão e rejeição (i. e., Fator I do IEP), o qual relaciona-se à expectativa de que as necessidades de conexão, segurança e estabilidade do sujeito não serão atendidas pelos outros (Lopes \& Melo, 2014). Esse domínio esquemático é comum em pacientes cujas famílias não satisfazem às necessidades básicas de aceitação e pertencimento (Wainer et al., 2016; Young et al., 2008), bem como ambientes familiares que apresentam características de frieza, instabilidade, rejeição e, inclusive, negligência emocional (Farrell et al., 2014).

Tabela 3

Fatores Indicados pela Paciente no IEP

\begin{tabular}{|c|c|c|}
\hline \multirow{2}{*}{ Fatores } & \multicolumn{2}{|c|}{ IEP } \\
\hline & Materno & Paterno \\
\hline FI: Desconexão e rejeição & $3,61^{*}$ & $3,39^{*}$ \\
\hline FII: Afetividade e estabilidade emocional & 2,11 & 1,33 \\
\hline FIII: Hipervigilância e orientação para o outro & $3,18^{*}$ & $3,36^{*}$ \\
\hline FIV: Superproteção e autonomia prejudicada & 1,00 & 1,00 \\
\hline FV: Limites prejudicados & 1,80 & 2,00 \\
\hline
\end{tabular}

Fonte. Primária (2015). ${ }^{*}$ Escores clinicamente relevantes

Semelhantemente, escores elevados no Fator III: Hipervigilância e orientação para o outro (escala materna: $m=3,18$; escala paterna: $m=3,36$ ) que, segundo Valentini et al. (2013), está associado a estilos parentais controladores, exigentes ou inflexíveis quanto a regras e preocupados com a opinião dos outros, também foram encontrados. Quanto à preocupação de Sônia em relação aos outros, Martha explica que "Ela era assim, muito influenciável, mas pelos outros; se viesse algum vizinho ou alguém falar qualquer coisa, era lei. Ela não ia lá verificar a veracidade nem nada. Simplesmente aquilo era verdade. Agora se nós, os filhos, falássemos alguma coisa, não! Nada tinha valor, nada era verdade, nada disso...”.

No que diz respeito ao protocolo do método de Rorschach no sistema compreensivo, verificou-se a presença do Índice de Déficit Relacional (CDI) positivo, indicando que Martha manifesta limitações importantes de enfrentamento em relação às demandas do dia a dia e do seu meio social. Embora manifeste relativo interesse no relacionamento com os outros, pode encontrar dificuldade em compreender bem as pessoas, a qual está associada a problemas expressivos de interação e à pouca necessidade 
das relações interpessoais (Nascimento, 2010). De uma maneira geral, o CDI positivo no Rorschach está fortemente vinculado à evitação de Martha no que se refere à formação e a manutenção dos seus relacionamentos pessoais, ocasionando prejuízos clinicamente relevantes para a paciente. Provavelmente, isso acontece porque Martha revela autoestima reduzida, conforme indicado no Rorschach, e não considera a si mesma, de modo suficiente, como foco de atenção (i. e., "eu sou diferente... [das outras pessoas]"), o que sugere também uma imagem pessoal desvalorizada (Nascimento, 2012).

Semelhantemente, revela capacidade reduzida em mobilizar os próprios recursos no enfrentamento das demandas do ambiente e no desenvolvimento de estratégias efetivas para lidar com o estresse, o que pode dificultar a sua adaptação a determinadas situações (Nascimento, 2012). Em alguns momentos, ainda, Martha também poderá apresentar pensamentos intrusivos constantes dos quais tem pouco controle, sugerindo a presença de um forte componente obsessivo de personalidade (Nascimento, 2012). Normalmente, essa característica indicada na avaliação da paciente por meio do método de Rorschach relaciona-se a uma estrutura obsessivo-compulsiva de personalidade descrita na literatura e, com frequência, associada ao TA (Schmidt et al., 2014).

No que se refere à maneira como processa e interpreta a informação do exterior, Martha demonstra atenção aos aspectos mais comuns e práticos do campo de estimulação e, na maioria das vezes, percebe a realidade de modo relativamente simples e adequado, isto é, sem grandes distorções perceptivas. Entretanto, em alguns momentos, poderá interpretar os fatos à sua própria maneira, mas não necessariamente patológica. De uma maneira geral, a participante prefere resolver problemas, tomar decisões e enfrentar situações de vida por meio do pensamento e da ideação racional, sem a interferência de aspectos emocionais (Villemor-Amaral \& Primi, 2012).

Quanto à modulação afetiva, Martha reflete uma tendência a controlar excessivamente suas descargas emocionais, dificilmente expressando-se afetivamente, o que confirma o seu relato de que raramente exterioriza as emoções. No entanto, assim como informado durante a entrevista, o protocolo também sugere a presença de sentimentos de hostilidade e ressentimento que podem interferir ativamente no seu ajustamento ao ambiente (Nascimento, 2010, 2012). Aparentemente, quando associadas à falta de recursos para lidar com as situações do dia a dia, a baixa tolerância ao estresse e a dificuldade em expressar as emoções apresentadas por Martha ocasionam potenciais prejuízos na sua adaptação, também comprometendo a sua interação com os outros e o ambiente.

\section{Considerações Finais}

O TA pode ocasionar significativo sofrimento e dificuldades de ajustamento nos diferentes âmbitos interpessoais. Os resultados da avaliação psicológica aplicados à paciente estudada não evidenciaram a presença de sintomas significativos de ansiedade, depressão e estresse, mas esquemas de privação emocional, isolamento social, inibição emocional e autossacrifício foram encontrados. Semelhantemente, vivências associadas ao domínio de desconexão e rejeição foram confirmadas pelo IEP, o QEY-S2 e a entrevista semiestruturada, sugerindo uma possível relação entre sintomas de TA, os EIDs e as experiências emocionais negativas vivenciadas pela paciente durante a infância. Ainda, os achados do Rorschach confirmaram o distanciamento interpessoal mencionado anteriormente.

Por tratar-se de um estudo de caso, uma limitação da presente pesquisa consiste na dificuldade da generalização dos dados encontrados em relação à população estudada. Nesse sentido, novos estudos com delineamento transversal são sugeridos no intuito de substanciar os resultados obtidos e também avaliar a relação entre os EIDs, domínios esquemáticos e o TA encontrados na presente investigação. Por fim, como alternativa de tratamento, acredita-se que pacientes com o diagnóstico estudado poderão se beneficiar da terapia focada em esquemas a fim de trabalhar aspectos relacionados à acumulação, à desordem e à dificuldade de descarte e sua associação com as questões de apego, bem como a experiências emocionais primitivas.

\section{Referências}

Albert, U., De Cori, D., Barbaro, F., Fernández de la Cruz, L., Nordsletten, A. E., \& Mataix-Cols, D. (2015). Hoarding disorder: A new obsessive-compulsive related disorder in DSM-5. Journal of Psychopathology, 21, 354-364. Recuperado de http://www.researchgate.net/ publication/285883585

American Psychiatric Association, APA. (2014). Manual diagnóstico e estatístico de transtornos mentais. 5. ed. Porto Alegre, RS: Artmed.

Araújo, Á. C., \& Lotufo Neto, F. (2014). A nova classificação americana para os transtornos mentais - o DSM-5. Revista Brasileira de Terapia Comportamental e Cognitiva, 16(1), 67-82. Recuperado de http://pepsic.bvsalud.org/scielo.php?pid=S1517$55452014000100007 \&$ script $=$ sci_arttext

Ayers, C. R., Najmi, S., Mayes, T. L., \& Dozier, M. E. (2014). Hoarding Disorder in older adulthood. The American Journal of Geriatric Psychiatry, 23(4), 416-422. doi: 10.1016/j.jagp.2014.05.009 
Brakoulias, V., \& Milicevic, D. (2015). Assessment and treatment of hoarding disorder. Australasian psychiatry: Bulletin of Royal Australian and New Zealand College of Psychiatrists, 23(4), 358-360. doi: 10.1177/1039856215587235

Brakoulias, V., Eslick, G. D., \& Starcevic, V. (2015). A meta-analysis of the response of pathological hoarding to pharmacotherapy. Psychiatry Research, 229(1), 272-276. doi: 10.1016/j.psychres.2015.07.019

Cazassa, M. J., \& Oliveira, M. S. (2012). Validação brasileira do Questionário de Esquemas de Young: Forma breve. Estudos de Psicologia (Campinas), 29(1), 23-31. doi: 10.1590/S0103-166X2012000100003

Cunha, J. (2001). Manual da versão em português das Escalas Beck. São Paulo, SP: Casa do Psicólogo.

Farrell, J. M., Reiss, N., \& Shaw, I. A. (2014). The schema therapy clinician's guide: A complete resource for building and delivering individual, group and integrated schema mode treatment programs. Malden, US: Wiley-Blackwell.

Fontenelle, L. F., \& Grant, J. E. (2014). Hoarding disorder: A new diagnostic category in ICD-11? Revista Brasileira de Psiquiatria, $36,28-39$. doi: 10.1590/1516-4446-2013-1269

Frías-Ibáñez, Á., Palma-Sevillano, C., Barón-Fernández, F., Bernáldez-Fernández, I., \& Aluco-Sánchez, E. (2014). Estatus nosológico de la acumulación compulsiva: ¿Subtipo de trastorno obsesivo-compulsivo o entidad clínica independiente?. Actas Esp. Psiquiatr., 42(3), 116-24. Recuperado de http://actasespanolasdepsiquiatria.es/repositorio/16/89/ESP/16-89-ESP-116-124-974335.pdf

Frost, R. O., \& Steketee, G. (Eds.). (2014). The Oxford handbook of hoarding and acquiring. New York, US: Oxford University Press.

Grisham, J. R., \& Baldwin, P. A. (2015). Neuropsychological and neurophysiological insights into hoarding disorder. Neuropsychiatric Disease and Treatment, 11, 951-962. doi: 10.2147/NDT.S62084

Hisatugo, C. L. C., \& Yazigi, L. (2014). Estudo exploratório com indivíduos com depressão por meio do Rorschach, Sistema Compreensivo. Avaliação Psicológica, 13(2), 157-166. Recuperado de http://pepsic.bvsalud.org/scielo.php?script=sci_arttext\&pid $=$ S1677-04712014000200003

Kress, V. E., Stargell, N. A., Zoldan, C. A., \& Paylo, M. J. (2016). Hoarding disorder: Diagnosis, assessment, and treatment. Journal of Counseling E Development, 94(1), 83-90. doi: 10.1002/jcad.12064

Lipp, M. E. N. (2000). Manual do Inventário de Sintomas de Stress para Adultos de Lipp (ISSL). São Paulo, SP: Casa do Psicólogo.

Lopes, J. B., \& Melo, W. V. (2014). A compreensão da repercussão dos esquemas iniciais desadaptativos, através da Teoria dos Esquemas, para otimização dos tratamentos de traumas. Saúde e Desenvolvimento Humano, 2(1), 75-86. Recuperado de http://sphinx.unilasalle.edu.br/ index.php/saude_desenvolvimento/article/view/1511/1101

Mataix-Cols, D. (2014). Hoarding disorder. New England Journal of Medicine, 370, 2023-2030. doi: 10.1056/NEJMcp1313051

Mathews, C. A. (2014). Hoarding disorder: More than just a problem of too much stuff. The Journal of Clinical Psychiatry, 75(8), 893-894. doi: 10.4088/JCP.14ac09325

Mihura, J. L., Meyer, G. J., Dumitrascu, N., \& Bombel, G. (2013). The validity of individual Rorschach variables: Systematic reviews and meta-analyses of the comprehensive system. Psychological Bulletin, 139(3), 548-605. doi: 10.1037/a0029406

Muroff, J., \& Underwood, P. (2016). Treatment of an adult with hoarding disorder. Em E. A. Storch, \& A. B. Lewin (Eds.), Clinical handbook of obsessive-compulsive and related disorders: A case-based approach to treating pediatric and adult populations (pp. 241-258). New York, US: Springer International Publishing. doi: 10.1007/978-3-319-17139-5

Muroff, J., Steketee, G., Frost, R. O., \& Tolin, D. F. (2014). Cognitive behavior therapy for hoarding disorder: Follow-up findings and predictors of outcome. Depression and Anxiety, 31(12), 964-971. doi: 10.1002/da.22222

Nascimento, R. S. G. F. (2010). Sistema Compreensivo do Rorschach: Teoria, pesquisa e normas para a população brasileira. São Paulo, SP: Casa do Psicólogo.

Nascimento, R. S. G. F. D. (2012). Estudo normativo para o Sistema Compreensivo do Rorschach. Avaliação Psicológica, 11(3), $361-374$. Recuperado de http://pepsic.bvsalud.org/scielo.php?script=sci_arttext\&pid=S1677-04712012000300005

Rasmussen, J., Steketee, G., Silverman, M., \& Wilhelm, S. (2013). The relationship of hoarding symptoms to schizotypal personality and cognitive schemas in an OCD sample. Journal of Cognitive Psychotherapy, 27(4), 384-396. doi: 10.1891/0889-8391.27.4.384

Schmidt, D. R., Della Méa, C. P., \& Wagner, M. F. (2014). Transtorno da acumulação: Características clínicas e epidemiológicas. Revista CES Psicología, 7(2), 27-43. Recuperado de http://revistas.ces.edu.co/index.php/psicologia/article/view/2895

Shaw, A. M., Timpano, K. R., Steketee, G., Tolin, D. F., \& Frost, R. O. (2015). Hoarding and emotional reactivity: The link between negative emotional reactions and hoarding symptomatology. Journal of Psychiatric Research, 63, 84-90. doi: 10.1016/j.jpsychires.2015.02.009

Shaw, A. M., Witcraft, S. M., \& Timpano, K. R. (2015). The relationship between traumatic life events and hoarding symptoms: A multimethod approach. Cognitive Behaviour Therapy, 1-11. doi: 10.1080/16506073.2015.1101150

Thompson, C., Cruz, L. F., Mataix-Cols, D., \& Onwumere, J. (2016). Development of a brief psychoeducational group intervention for carers of people with hoarding disorder: A proof-of-concept study. Journal of Obsessive-Compulsive and Related Disorders, 9, 66-72. doi: 10.1016/j.jocrd.2016.03.002

Timpano, K. R., Shaw, A. M., Cougle, J. R., \& Fitch, K. E. (2014). A multifaceted assessment of emotional tolerance and intensity in hoarding. Behavior Therapy, 45(5), 690-699. doi: 10.1016/j.beth.2014.04.002

Tolin, D. F., Frost, R. O., Steketee, G., \& Muroff, J. (2015). Cognitive behavioral therapy for hoarding disorder: A meta-analysis. Depression and anxiety, 32(3), 158-166. doi: 10.1002/da.22327

Valentini, F., \& Alchieri, J. C. (2009). Modelo clínico de estilos parentais de Jeffrey Young: Revisão da literatura. Contextos Clínicos, 2(2), 113123. Recuperado de http://pepsic.bvsalud.org/scielo.php?pid=S1983-34822009000200006\&script=sci_arttext

Valentini, F., Alchieri, J. C., \& Laros, J. A. (2013). Validity evidence for the reduced version of the Young Parenting Inventory (YPI). Paideia, 23(56), 293-300. doi: 10.1590/1982-43272356201303

Van Genderen, H., Rijkeboer, M., \& Arntz, A. (2012). Theoretical Model: Schemas, Coping Styles, and Modes. Em M. Van Vreeswijk, J. Broersen, \& M. Nadort (Eds.), The Wiley-Blackwell handbook of schema therapy: Theory, research and practice (pp. 27-40). Chichester, Reino Unido: John Wiley \& Sons.

Villemor-Amaral, A. E., \& Primi, R. (2012). Teste de Zulliger no Sistema Compreensivo - ZSC: forma individual. São Paulo, SP: Casa do Psicólogo.

Wainer, R., Paim, K., Erdos, R., \& Andriola, R. (2016). Terapia cognitiva focada em esquemas: Integração em psicoterapia. Porto Alegre, RS: Artmed.

Wheaton, M. G. (2016). Understanding and treating hoarding disorder: A review of cognitive-behavioral models and treatment. Journal of Obsessive-Compulsive and Related Disorders, 9, 43-50. doi: 10.1016/j.jocrd.2016.02.006 
Yin, R. K. (2015). Estudo de caso: Planejamento e métodos. 5. ed. São Paulo, SP: Bookman editora.

Young, J. E., Klosko, J. S., \& Weishaar, M. E. (2008). Terapia do esquema: Guia de técnicas cognitivo-comportamentais inovadoras. Porto Alegre, RS: Artmed.

\section{Sobre os autores}

Diego Rafael Schmidt é psicólogo, Especialista em Avaliação e Diagnóstico Psicológico: Enfoque clínico pela Faculdade Meridional/ IMED. Possui formação internacional em hipnoterapia ericksoniana pelo ACT Institute.

Cristina Pilla Della Méa é Psicóloga, Mestre em Envelhecimento Humano pela Universidade de Passo Fundo (UPF). Docente do Curso de Psicologia da IMED. 